\title{
Protective Effects of Forsythiaside A, Forsythiaside B, and Phillyrin against UVA-Induced Cell Damage
}

\author{
Fu-Jun Jin ${ }^{1}$, Hong-Wei Zhao ${ }^{2}$, Xiao Yuan ${ }^{1}$, Zhi-Yun Wu ${ }^{2}$, Qiao-Li Wang ${ }^{1}$, Chung-Wah Ma ${ }^{2}$, Zhe Ren ${ }^{1, *}$, \\ Yi-Fei Wang,* \\ ${ }^{1}$ Guangzhou Jinan Biomedicine Research and Development Center, National Engineering Research Center of Genetic Medicine, Jinan \\ University, Guangzhou, PR China \\ ${ }^{2}$ Infinitus (China) Company Co., Ltd, Guangzhou, PR China \\ *Corresponding author: rz62@163.com; twang-yf@163.com
}

Received August 11, 2014; Revised August 26, 2014; Accepted September 04, 2014

\begin{abstract}
Chronic exposure to ultraviolet (UV) radiation affects diverse constituents of skin and induces photoaging. Current therapeutic approaches cannot fully reverse the pathophysiology of photoaging, thus necessitating the discovery of new strategies to protect skin from UV radiation. Natural products have long been reported to possess photoprotective effects. Herein, we investigated the potential anti-UVA effects of forsythiaside A, forsythiaside B, and phillyrin extracted from Forsythia suspensa (Thunb.) Vahl. In vitro, all three compounds significantly prevented cytotoxicity, DNA damage, apoptosis, and cell-cycle arrest caused by a low dose of UVA. All three compounds also inhibited the expression of inflammatory cytokines. Collectively, our results suggest that these three compounds could be preventives against UVA-induced skin damage.
\end{abstract}

Keywords: Forsythiaside A, Forsythiaside B, Phillyrin, Anti-UVA, Anti-inflammatory

Cite This Article: Fu-Jun Jin, Hong-Wei Zhao, Xiao Yuan, Zhi-Yun Wu, Qiao-Li Wang, Chung-Wah Ma, Zhe Ren, and Yi-Fei Wang, "Protective Effects of Forsythiaside A, Forsythiaside B, and Phillyrin against UVAInduced Cell Damage.” Journal of Food and Nutrition Research, vol. 2, no. 9 (2014): 587-593. doi: 10.12691/jfnr-2-9-10.

\section{Introduction}

Aging is one of the most elementary biological constants, ultimately culminating in the inability to adapt to metabolic stress and a decline of biological function [1]. The skin is the largest organ of the body and is a physiological barrier against damaging environmental effects. Skin aging is characterized as either intrinsic or extrinsic aging senescence, with the latter mainly due to environmental factors like UV radiation, smoking, wind, sun, harmful chemicals $[2,3]$.

Photoaging is induced by chronic skin exposure to solar UV radiation and is a critical initiator of many skin diseases [4]. Ultraviolet rays damage the skin by causing oxidative stress, inflammation, DNA damage, production of proteolytic enzymes, immunosuppression, and stimulation of growth factors that promote cancer cell growth $[5,6,7]$. The clinical symptoms of skin photoaging include mottled dyspigmentation, wrinkling, sagging, fragility, easy bruising, loss of elasticity, accumulation of precancerous lesions, and epithelial neoplasms [5]. Solar rays can be divided into long wave (UVA; $320-400 \mathrm{~nm}$ ), mid wave (UVB; 290-320 nm) and short wave (UVC; 200-290 nm) [8]. UVC radiation is strongly absorbed by the ozone layer, barely reaching the earth's surface. UVB radiation constitutes only $5 \%$ of total solar UV radiation but is primarily responsible for skin cancers and other skin diseases [9]. The remaining $90-95 \%$ of the solar UV spectrum is UVA, which can penetrate deep into our dermis and epidermis. UVA is responsible for chronic damage associated with photoaging as well as malignant cancers [10]. As the ozone layer is progressively destroyed, more UV radiation reaches the earth, intensifying photoaging. New UV-protective agents are therefore urgently needed to provide increased skin protection.

Forsythia suspensa (Thunb.) Vahl, is a widely distributed climbing plant and an important traditional Chinese medicine [11]. It has significant antiviral, antibacterial, antioxidant, and anti-inflammatory activities $[12,13]$. Lignans, phenylethanoid glycosides, flavonoids, volatile oils, and terpenes have all been extracted from the plant, among which forsythiaside A, forsythiaside B, and phillyrin are thought to be the main bioactive constituents. Forsythiasides A and B have potent neuroprotective and cardioprotective effects $[14,15,16]$. To our knowledge, however, UV-protective activity has not been reported for these compounds. We therefore sought to evaluate whether these compounds might protect against UVAinduced skin damage.

\section{Materials and Methods}

\subsection{Chemicals}


TRIzol® Reagent, low-melting-point agar, and regular agar were obtained from Invitrogen (Invitrogen, CA, USA). 2-(2, 5-dimethyl-2-thiazolyl)-2, 5-diphenyl-2Htetrazolium bromide (MTT), lipopolysaccharide (LPS), propidium iodide (PI), and annexin V-FITC were purchased from Sigma-Aldrich (Sigma-Aldrich, MO, USA). Fetal bovine serum (FBS), Dulbecco's modified Eagle medium (DMEM), and penicillin-streptomycin were bought from Gibco (Gibco, CA, USA). All other reagents used were of pharmaceutical grade.

\subsection{Cell Culture}

NIH/3T3 fibroblast cells and RAW 264.7 macrophages were obtained from Cell Bank of the Chinese Academy of Sciences, Shanghai, PR China and were cultured in DMEM supplemented with $10 \%$ heat-inactivated FBS and $100 \mathrm{U} / \mathrm{mL}$ penicillin/streptomycin in a humidified incubator with a $5 \% \mathrm{CO}_{2}$ atmosphere at $37^{\circ} \mathrm{C}$.

\subsection{Preparation of Phillyrin, Forsythiaside A, and Forsythiaside $B$}

$250 \mathrm{~g}$ of $F$. suspense was ground into 30-60 mesh powder, extracted with 2000 g 70\% ethanol, and extracted again with $1500 \mathrm{~g} 70 \%$ ethanol. The combined extracts were filtered and evaporated, and the residue was suspended in a 2-fold weight ratio of water and loaded onto a $0.4 \mathrm{~L}$ macropore resin PIPO-02 column (C1). C1 was washed with purified water until the eluate became light in color, and a second PIPO-02 column (C2) was connected. The columns were washed with 20-30 column volumes of methanol/ethyl acetate (4:1): $0.2 \%$ formic acid (5:98), after which a third PIPO-02 column (C3) was connected and washed with methanol/ethyl acetate (4:1): $0.2 \%$ formic acid (10:90) until forsythiaside $\mathrm{A}$ was detected in the eluate. $\mathrm{C} 1$ was then disconnected, and a 70 $\mathrm{mL}$ C-18 column was connected to C3 and eluted with methanol/ethyl acetate (4:1): $0.2 \%$ formic acid (10:90) until forsythiaside A was detected in the eluate. The C18 column was then disconnected from C3 and washed with methanol. After evaporating the fractions, the purity of forsythiaside A was $98 \%$. Next, a second C18 column was connected to column 3 and washed with methanol/ethyl acetate (4:1): 0.2\% formic acid (10:90) until forsythiaside $\mathrm{B}$ was detected in the eluate. The $\mathrm{C}-18$ column was then disconnected from C3 and washed with methanol. The collected methanol fractions were evaporated and refrigerated to yield forsythiaside B at 98\% purity. Finally, column C1 was connected to another PIPO-02 column (C4) and eluted with methanol/ethyl acetate (4:1): 0.2\% formic acid (15:85) and then eluted with methanol/ethyl acetate (4:1): $0.2 \%$ formic acid (20:80). The combined eluate fractions were concentration and crystallized, and the raw crystals were recrystallized in $70 \%$ ethanol to yield phillyrin at $98 \%$ purity. The identity and purity of each compound were confirmed by HPLC and by comparison to known standards supplied from PI \& PI Biotech Inc.

\subsection{Cytotoxicity Assays}

The cytotoxicity of forsythiaside A, forsythiaside B, and phillyrin toward NIH/3T3 fibroblast cells and RAW 264.7 macrophages was determined using MTT assays.
Cells were plated in 96-well plates in $100 \mu \mathrm{L}$ growth media (DMEM with 10\% FBS), cultured overnight, and exposed to a range of compound concentrations for $48 \mathrm{~h}$. $10 \mu \mathrm{l}$ of a $5 \mathrm{mg} / \mathrm{mL}$ MTT stock solution was added to each well, and the plate was incubated for $4 \mathrm{~h}$ in the dark. The MTT solution was discarded and $100 \mu \mathrm{L}$ dimethyl sulfoxide (DMSO) was added to each well. Plates were gently shaken for $15 \mathrm{~min}$ at room temperature, after which the OD at 570 and $630 \mathrm{~nm}$ were measured with an enzyme immunoassay reader (EIA) (Bio-Rad USA). The normalized cell viability for each compound concentration was determined by comparison to a compound-free control.

\subsection{UVA Irradiation}

NIH/3T3 fibroblast cells were grown in 96-well plates to $60-70 \%$ confluence, after which the medium was discarded, and the cells were covered with a thin layer of phosphate-buffered saline (PBS). Plates were then treated with $1.4 \mathrm{~J} / \mathrm{cm}^{2} \mathrm{UVA}$ for 15,30 , 45, or $70 \mathrm{~min}$ generated from a model HB 406/A Philips Original Home Solarium (Philips, Groningen, Holland), and the dose was confirmed with a UV Power Pack Radiometer (EIT Inc., Sterling, VA). PBS was then removed and replaced with growth medium, and the plates were cultured for another 24 h. As a control, several plates were mock treated without radiation. $48 \mathrm{~h}$ after UVA irradiation the viability of cells in each group was measured by MTT. The $50 \%$ cytotoxic irradiation dose $\left(\mathrm{CC}_{50}\right)$ was calculated and used in subsequent UVA irradiation experiments.

\subsection{Evaluation of UVA Protective Activity of each Compound}

NIH/3T3 fibroblast cells were grown in 96-well plates to $60-70 \%$ confluence and pretreated for $24 \mathrm{~h}$ with the indicated concentrations of each compound in growth medium. The medium was then replaced by sterile PBS, and the plates were treated with a CC50 UVA dose. PBS was then removed and replaced with growth medium without compound. As a comparator, a control plate received UVA exposure without compound pretreatment. $24 \mathrm{~h}$ after UVA irradiation the cell viability of each group was measured by MTT assay and calculated in comparison to the control.

\subsection{Comet Assay}

NIH/3T3 fibroblast cells were grown in 12-well plates to $60-70 \%$ confluence. The medium was discarded and replaced with $1 \mathrm{~mL}$ of growth medium per well that contained the standardized test dose of each compound: $100 \mu \mathrm{g} / \mathrm{mL}$ forsythiaside $\mathrm{A}, 6.25 \mu \mathrm{g} / \mathrm{mL}$ forsythiaside $\mathrm{B}$, or $200 \mu \mathrm{g} / \mathrm{mL}$ phillyrin. The plates were cultured for another $24 \mathrm{~h}$ and treated with the CC50 UVA dose for 24 h. The cells were digested, collected by centrifugation, and suspended in PBS at a concentration of $1 \times 10^{6}$ cells/mL. Cell suspensions were mixed with $0.8 \%$ lowmelting-point agar and placed onto a slide precoated with $0.7 \%$ regular agar. After the agar solidified, slides were soaked in a prechilled, fresh lysing solution $(\mathrm{pH} 10)$ for $1.5-2 \mathrm{~h}$ at $4^{\circ} \mathrm{C}$. After rinsing with fresh $0.4 \mathrm{M}$ Tris buffer ( $\mathrm{pH} 7.5)$, slides were soaked for 15 minutes in a reservoir filled with electrophoresis buffer $(\mathrm{pH}>13)$ and subjected 
to electrophoresis for another $15 \mathrm{~min}(25 \mathrm{~V}, 300 \mathrm{~mA})$. Slides were neutralized three times by a 5 min soak with Tris-HCl (pH 7.5). Slides were then stained with PI and photographed under a fluorescent microscope (OLYMPUS IX71). The percentages of tail DNA, tail length, and Oliver tailMor, which indicates damaged DNA, were analyzed using the Casp software program (version 1.2.2).

\subsection{Apoptosis and Cell cycle Analysis}

NIH/3T3 fibroblasts were grown in a 6-well cell culture plate to $60-70 \%$ confluency and treated with growth medium containing the standardized test dose of each compound for $24 \mathrm{~h}$. The plates were then exposed to 1.4 $\mathrm{J} / \mathrm{cm}^{2} \mathrm{UVA}$ for $45 \mathrm{~min}$. After another $24 \mathrm{~h}$, the cells were harvested in cold PBS, fixed in $70 \%$ ethanol, and stored overnight at $4^{\circ} \mathrm{C}$. The cells were washed once with PBS and resuspended in $1 \mathrm{~mL}$ of $50 \mathrm{mg} / \mathrm{mL}$ PI staining reagent containing $100 \mu \mathrm{g} / \mathrm{mL}$ RNase, then incubated in the dark for $30 \mathrm{~min}$. The percentage of cells in each cell cycle phase was measured by fluorescence activated cell sorting (FACS). For apoptosis analysis, the cells were harvested and washed twice with cold PBS, after which they were resuspended in $100 \mu \mathrm{L}$ incubation buffer with annexin VFITC and PI. After incubation in the dark for $15 \mathrm{~min}$, apoptosis was analyzed by FACS.

\subsection{LPS-stimulated Inflammation Assay}

RAW 264.7 macrophages were cultured in 6-well plates to $60-70 \%$ confluence. The medium was discarded and cells were washed twice with PBS. Serum-free DMEM medium was added to each well. After $24 \mathrm{~h}$, the medium was replaced with growth medium that contained the standardized test dose of each compound, and the plates were incubated for $4 \mathrm{~h}$. The medium was then replaced with LPS induction medium (100 ng/mL) and cultured for another $24 \mathrm{~h}$, after which the cells were collected for total RNA isolation.

\subsection{RNA Isolation, Reverse Transcription, and Quantitative Real-time PCR (qPCR)}

Total RNA was extracted using TRIzol ${ }^{\circledR}$ Reagent, and RNA concentrations were measured using a spectrophotometer (Thermo, USA) monitoring at 260 and $280 \mathrm{~nm}$. $500 \mathrm{ng}$ of extracted RNA was reverse transcribed using a PrimeScript RT reagent kit (Takara, Japan), and qRT-PCR assays were conducted using SsoFast ${ }^{\mathrm{TM}}$ EvaGreen $^{\circledR}$ Supermix (Bio-Rad, USA) according to the manufacturer's instructions. Primer pairs used were specific for IL-1 $\beta$ (F: 5'-GAG CCT GTG TTT CCT CCT TG-3' and R: 5'-CAA GTG CAA GGC TAT GAC CA-3' ), IL-6 (F: 5'-CTG ACA ATA TGA ATG TTG GG-3' and R: 5'-TCC AAG AAA CCA TCT GGC TAG G-3' ), TNF- $\alpha$ (F: 5'-GGG AGC AAA GGT TCA GTG AT-3' and R: 5'CCT GGC CTC TCT ACC TTG TT-3' ), iNOS (F: 5'AAG CAG CTG GCC AAT GAG-3' and R: 5'-CCC CAT AGG AAA AGA CTG CA-3' ), MCP-1 (F: 5'-GTC TCT GCA ACG CTT CTG TGC C-3' and R: 5'-AGT CGT GTG TTC TTG GGT TGT GG-3' ), GAPDH (F: 5'-GTC ATT GAG AGC AAT GCC AG-3' and R: 5'-GTG TTC CTA CCC CCA ATG TG-3' ). The relative expression of each genes was normalized to the housekeeping gene GAPDH.

\subsection{Statistical Analysis}

Data were analyzed using GraphPad Prism 5 (GraphPad Software, La Jolla, CA), and results given are the mean \pm SEM. The statistical significance of specific comparisons was determined by the Student's t test; $p$ values of less than 0.05 were considered significant.

\section{Results}

\subsection{Cytotoxicity and UVA-protective Activity of $F$. suspensa Bioactives}

Forsythiaside A, forsythiaside B, and phillyrin were isolated from $F$. suspensa, and their chemical structures were unequivocally identified (Figure 1). The cytotoxicity of each was determined in NIH/3T3 cells by MTT assay. The maximal concentration at which no cytotoxicity was observed were $>200 \mu \mathrm{g} / \mathrm{mL}$ forsythiaside A, $50 \mu \mathrm{g} / \mathrm{mL}$ forsythiaside B, and > $200 \mu \mathrm{g} / \mathrm{mL}$ phillyrin (Figure $2 \mathrm{~A}$ ).
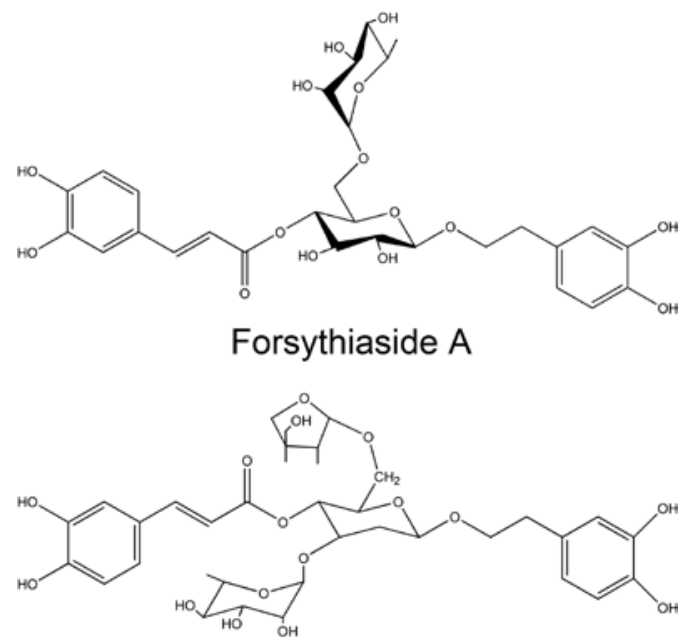

Forsythiaside B

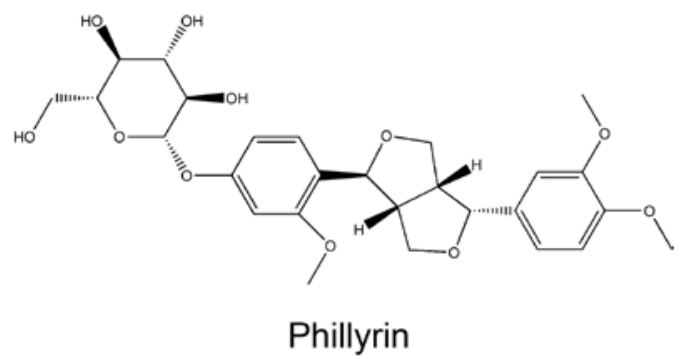

Figure 1. Chemical structures of Forsythiaside A, forsythiaside B, and phillyrin extracted from $F$. suspensa

To specially measure the UVA-protective activity of each compound on the dermis damage caused by UVA irradiation. The mouse fibroblast NIH/3T3 cells were chosen as a model of the dermis and be used in the sequent experiment. Firstly, we measured the cytotoxicity of irradiation with $1.4 \mathrm{~J} / \mathrm{cm}^{2} \mathrm{UVA}$ for $15,30,45$, and 70 min, followed by MTT assay for viability. The $45 \mathrm{~min}$ dose showed half maximal inhibition (Data not shown) and was used for UVA-protection assays. Next, the UVAprotective activity of each compound was measured after 
pretreatment with compounds and UVA exposure. All three compounds exhibited excellent UVA-protective activity (Figure 2B). Compared to a 50\% viability reduction in the absence of compound, viability was only decreased by $25 \%, 29 \%$, and $32 \%$ when cells were pretreated with $100 \mu \mathrm{g} / \mathrm{mL}$ forsythiaside A, $6.25 \mu \mathrm{g} / \mathrm{mL}$ forsythiaside $\mathrm{B}$, or $200 \mu \mathrm{g} / \mathrm{mL}$ phillyrin, respectively. These concentrations were selected as a standardized test concentration for further characterizations.
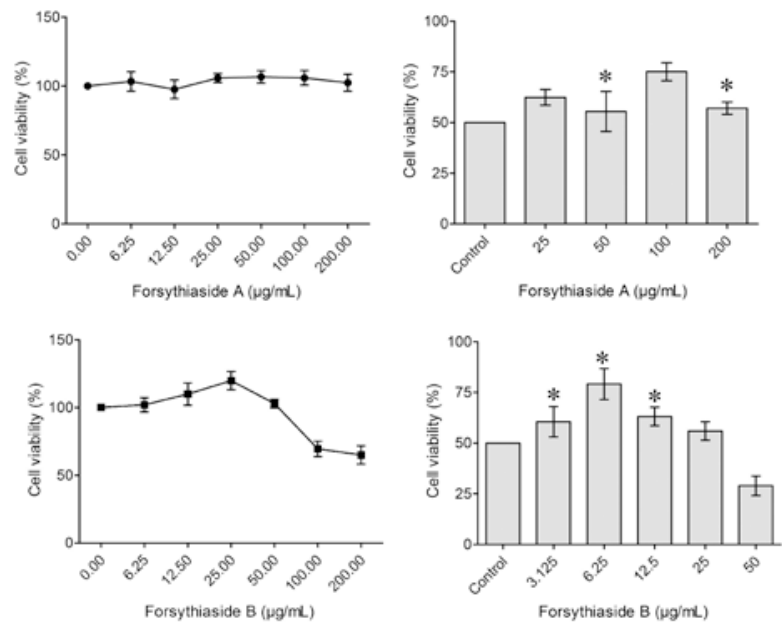

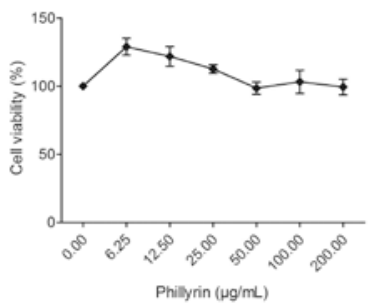

A

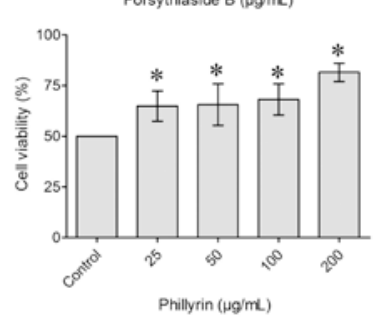

B
Figure 2. The UVA-protective activity of $F$. suspensa bioactives. (A) The cytotoxic activity of the compounds on NIH/3T3 cells. (B) The protective activity of a $24 \mathrm{~h}$ pretreatment with each compound against 48 h UVA exposure. Data are the mean values of at least three independent experiments \pm SEM. ${ }^{*} p<0.05$ : significant difference compared to the control group

\subsection{F. suspensa Bioactives Protect NIH/3T3 Cells from UVA Induced DNA Damage}

UVA irradiation can cause DNA damage by generating reactive oxygen species that form DNA adducts, cause double-strand breaks (DSBs), and make interstrand DNA crosslinks [17]. UVA irradiation caused severe DNA damage in NIH/3T3 cells in the absence of a protective compound, as evidenced by the appearance of comet tails of broken DNA after single-cell gel electrophoresis (Figure 3). In contrast, pretreatment with the standard test concentration of each compound $(100 \mu \mathrm{g} / \mathrm{mL}$ forsythiaside A, $6.25 \mu \mathrm{g} / \mathrm{mL}$ forsythiaside $\mathrm{B}$, or 200 $\mu \mathrm{g} / \mathrm{mL}$ phillyrin) for $24 \mathrm{~h}$ greatly decreased UVA-induced DNA damage. In fact, the percentage of DNA with tails decreased by $\sim 94 \%$, and the tail length reduced $89 \%$. The oliver tailMor also dropped to $89 \%$ after pretreatment with forsythiaside A. Quantitatively, forsythiaside B inhibited the formation of DNA with tails, tail length, and the oliver tailMor by $88 \%, 87 \%$, and $95 \%$, respectively. Similarly, phillyrin inhibited the three measurable by $74 \%, 73 \%$, and $89 \%$.

\subsection{F. suspensa bioactives Inhibit UVA- Induced Apoptosis and G1 Cell Cycle Arrest}

We next evaluated whether the $F$. suspensa bioactives could protect against UVA-induced apoptosis and cell cycle arrest using annexin-V and PI staining after exposure to UVA for $24 \mathrm{~h}$ in the presence of absence of the compounds at their standard test concentrations. As shown in Figure 4, the proportion of apoptosed cells increased $3.86 \%$ after low dose UVA irradiation in the absence of compound treatment, whereas no significant increase in apoptosis was observed after compound pretreatment. By flow cytometry, UVA irradiation increased the proportion of cells in $\mathrm{G} 1$ phase by $\sim 38 \%$ but only by $20 \%, 6.3 \%$, and $9.4 \%$ after pretreatment with each bioactive. Collectively, these results demonstrated a protective effect against apoptosis and G1 cell-cycle arrest.

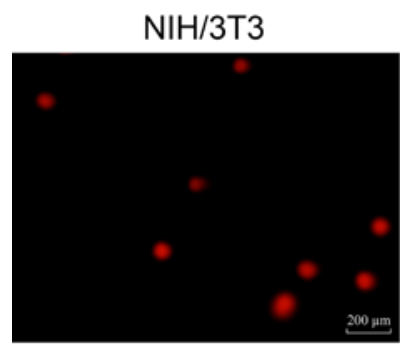

Forsythiaside A

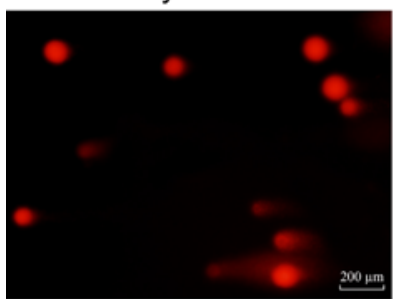

Phillyrin
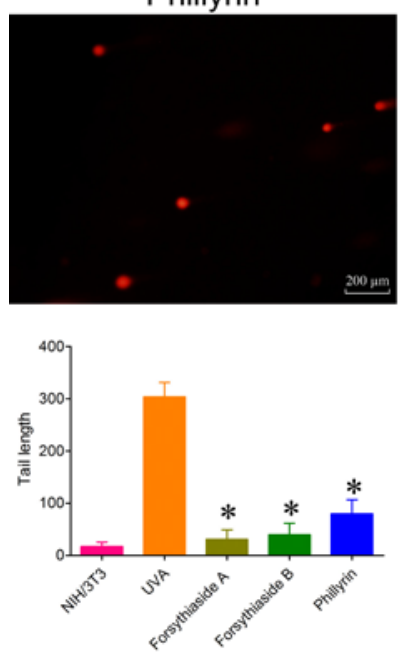

Figure 3. The protective activity of $F$. suspensa bioactives against DNA damage. NIH/3T3 cells were pretreated for $24 \mathrm{~h}$ with $100 \mu \mathrm{g} / \mathrm{mL}$ forsythiaside A, $6.25 \mu \mathrm{g} / \mathrm{mL}$ forsythiaside $\mathrm{B}$, or $200 \mu \mathrm{g} / \mathrm{mL}$ phillyrin followed by exposure to UVA for $24 \mathrm{~h}$, followed by single-cell electrophoretic assay of cells from each group. The percentages of tail DNA, tail length and oliver tailMor were analyzed using the Casp software program. Data shown are the mean values of at least three independent experiments \pm SEM. ${ }^{*} p<0.05$ : significant difference compared to the control group

\subsection{Anti-inflammatory Effect of $F$. suspensa Bioactives}


One of the principal mechanisms by which UVA irradiation causes cell damage is by inducing inflammation. To determine the anti-inflammatory activity of our compounds, we challenged RAW 264.7 cells with LPS in the presence or absence of compound treatment, and the expression levels of IL-1, IL-6, TNF- $\alpha$, MCP-1, and iNOS cytokines were monitored by qRT-PCR. Importantly, we confirmed the lack of cytotoxicity for each compound against RAW 264.8 cells, maximal nontoxic concentrations of forsythiaside A, forsythiaside $\mathrm{B}$, and phillyrin that were $>200 \mu \mathrm{g} / \mathrm{mL}, 50 \mu \mathrm{g} / \mathrm{mL}$ and $200 \mu \mathrm{g} / \mathrm{mL}$, respectively (Figure 5A). We then found that $100 \mu \mathrm{g} / \mathrm{mL}$ forsythiaside A significantly inhibited MCP-1 and iNOS expression by $60 \%$ and $23 \%$ (Figure 5B). Likewise, $6.25 \mu \mathrm{g} / \mathrm{mL}$ forsythiaside B inhibited MCP-1 expression by $56 \%$, and finally, pretreatment with 200 $\mu \mathrm{g} / \mathrm{mL}$ phillyrin reduced the expression of IL-1, IL-6, MCP-1, and TNF- $\alpha$ by $41 \%, 77 \%, 70 \%$, and $53 \%$, respectively. Thus, all of the $F$. suspensa bioactives exhibited significant anti-inflammatory activity.
A

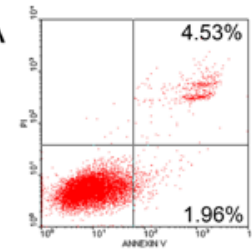

$\mathrm{NIH} / 3 \mathrm{~T} 3$

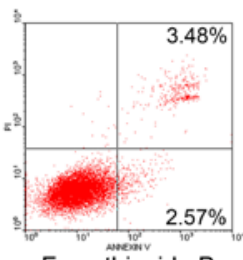

Forsythiaside B

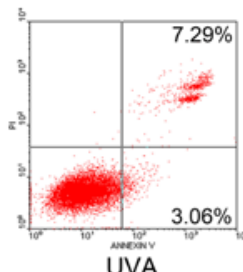

UVA
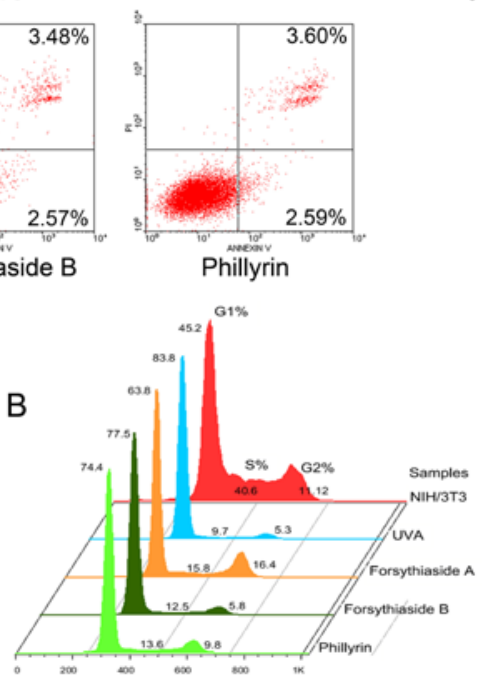

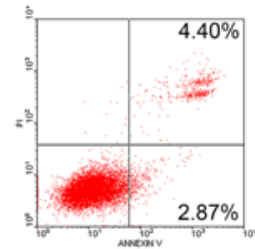

Forsythiaside A

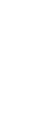

A

The majority of UV damage to skin cells is thought to occur via an oxygen-dependent mechanism involving multiple cellular targets, including proteins, membranes, and DNA [20]. The genotoxicity of UVA most likely occurs via indirect mechanisms that involve reactive oxygen species like superoxide anion ( $\left.2^{-}\right)$, hydroxyl $\left(\mathrm{OH}^{-}\right)$, and hydrogen peroxide $\left(\mathrm{H}_{2} \mathrm{O}_{2}\right)$ [21,22]. The damage caused includes pyrimidine dimers and double- or single-strand breaks, which can be removed by nucleotide excision repair (NER) or recombinational repair [23,24]. In many cases, cells will arrest at the G1 phase to enable DNA repair, thereby protecting cells against the propagation of mutations [25], and cell cycle regulators like p53, p21 play an important role in this response [26,27].

A
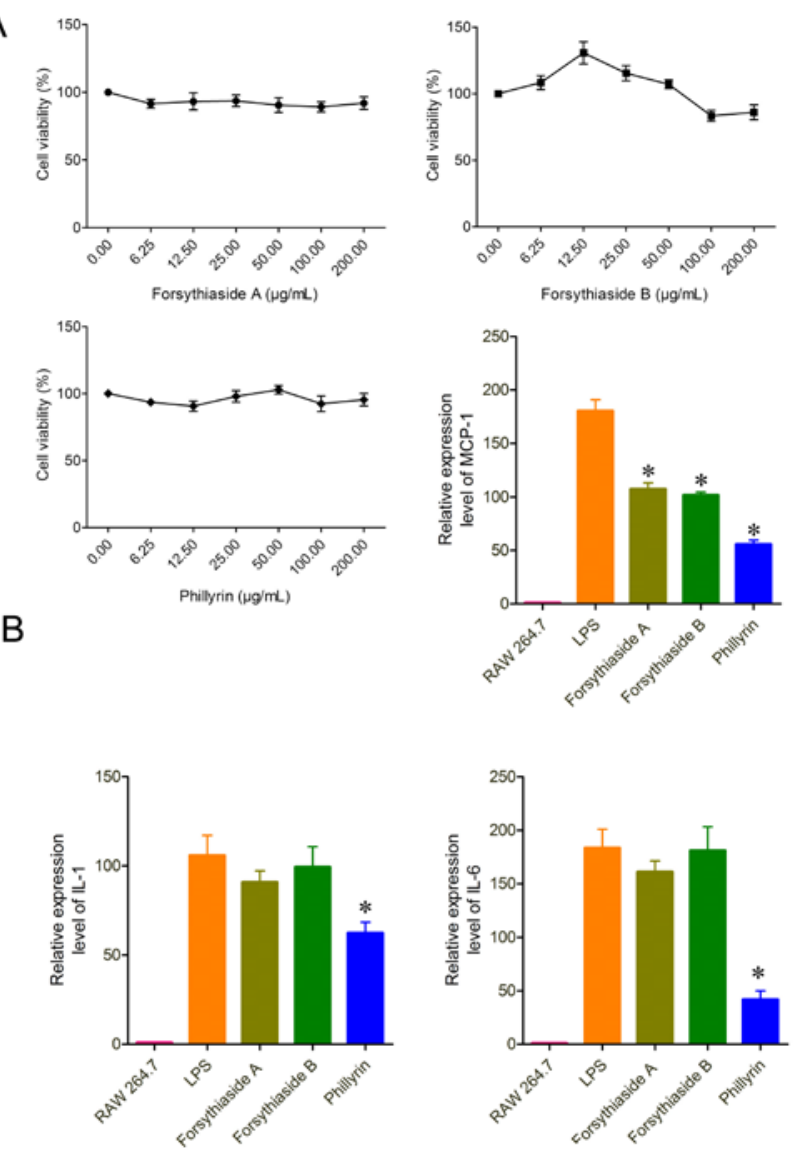

Figure 4. The protective effect of $F$. suspensa bioactives against UVAinduced apoptosis and cell-cycle arrest. (A) After exposure to UVA for 24h, untreated cells or cells pretreated as described in Figure 3 were irradiated, followed by staining with annexin-V/PI and quantification of apoptosis by FACS. (B) To assess cell-cycle arrest, the cells were stained with PI staining reagent, and the percentages of cells in each phase of the cell cycle were measured by FACS

\section{Discussion}

Photoaging is caused by chronic exposure to solar radiation and is a complex biological process that affects diverse skin constituents [18]. UV irradiation can trigger DNA damage, depletion of the antioxidant defense system, release of pro-inflammatory mediators, cell-cycle arrest, apoptosis, and immunosuppression [19]. The use of chemopreventive agents, especially natural products like plant polyphenols, has shown some effectiveness for preventing the damaging effects of UV irradiation [10].

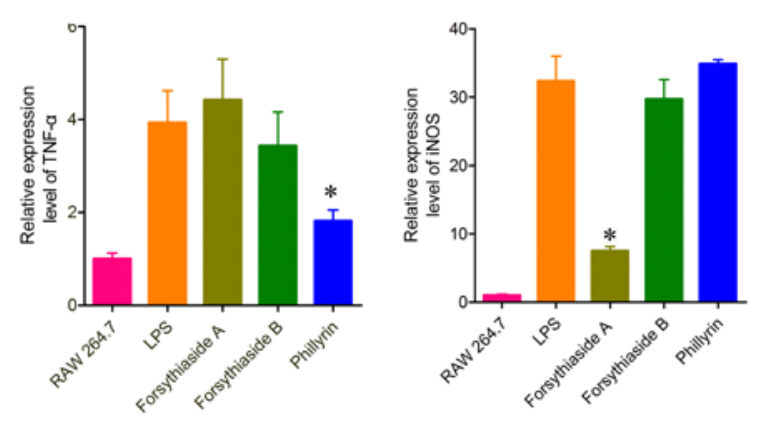

Figure 5. The anti-inflammatory activity of F. suspensa bioactives. (A) The cytotoxicity of the tested compounds toward RAW 264.7 cells. (B) Relative mRNA expression levels of IL- 1 , IL-6, TNF- $\alpha$, MCP-1, and iNOS after compound pretreatment and LPS stimulation. Compound concentrations were the same as in Figs. 3 and 4. The data presented are the average values from at least three independent experiments \pm SEM. ${ }^{*} p<0.05$ indicates a significant difference compared to the control group 
DNA damage caused by ROS and lipid peroxidation can also induce apoptosis, with ROS being the driver of UVA-induced apoptosis. This occurs by ROS repressing the expression of anti-apoptosis genes like bcl-2 and promoting the expression of apoptosis-related genes like p53 and c-myc [28]. Herein, we showed that a low dose of UVA irradiation was cytotoxic toward NIH/3T3 cells, caused significant DNA damage, and induced apoptosis and cell-cycle arrest.

We also evaluated the ability of three $F$. suspensa bioactive components, phillyrin, forsythiaside $\mathrm{A}$, and forsythiaside $\mathrm{B}$, to protect against these UVA-induced effects. For each determination, cells were pretreated with a non-toxic concentration of each compound and then exposed to a damaging dose of UVA. We found that all three bioactives were protective against UVA cytotoxicity. This protective effect is consistent with literature reports that these three compounds have antioxidative activity $[14,15,16]$.

Inflammation is also thought to be an important trigger of the pathogenesis of multiple age-related diseases including atherosclerosis, autoimmune disorders, and neurodegenerative diseases [29]. Some studies have even reported that inflammatory response to UVA exposure causes protein denaturation, DNA damage, and oxidative stress [30]. Macrophages are an important trigger of this response by secreting inflammatory mediators when activated [31]. We therefore evaluated whether the $F$. suspensa bioactives have anti-inflammatory activity in vitro with LPS-challenged RAW 264.7 cells. We found that phillyrin greatly inhibited the LPS-stimulated expression of IL-1, IL-6, TNF- $\alpha$, and MCP-1. Similarly, forsythiaside A significantly inhibited the LPS-stimulated expression of MCP-1 and iNOS, and forsythiaside B significantly inhibited the expression of MCP-1. These results are consistent with a previous reports in which these three compounds inhibited inflammation by suppressing activation of the NF- $\mathrm{BB}$ and MAPK pathways $[14,15,16,32]$. A series of studies over the past decade have also shown that chronic oxidative stress can cause activation of a variety of transcription factors such as NF$\kappa \mathrm{B}$ and $\mathrm{p} 53$, which may explain the anti-inflammatory activity of the compounds [33].

\section{Conclusions}

In conclusion, as our environment continues to change and increase our exposure to UVA irradiation, photoaging is becoming a more serious problem. Consequently, it is becoming more important to develop new preventive and therapeutic strategies against UVA skin damage. Herein, we showed that forsythiaside $\mathrm{A}$, forsythiaside $\mathrm{B}$, and phillyrin significantly attenuate cell damage caused by UVA and that this protective effect is characterized by protection against DNA damage, apoptosis, and G1 phase cell-cycle arrest. Moreover, the compounds significantly repress the expression of multiple inflammatory cytokines. With these promising activities and low inherent cytotoxicity, these compound may be promising and safe chemical additives for sunscreens.

\section{Acknowledgement}

This work was supported by the Key Projects in the National Science \& Technology Pillar Program during the Twelfth Five-year Plan Period (SQ2011SF12B02099), the Guangzhou Science and Technology Plan Projects (2013J4500058), the Guangdong University Scientific, and Technological Achievements of Major Projects (cgzhzd0905).

\section{Conflict of Interest}

The authors have no competing interests

\section{References}

[1] Young, J.C., "Physical dimensions of aging, 2nd edition”. Am. J. Hum. Biol 18. 156-158. 2006.

[2] Kurban, R.S., and Bhawan, J., "Histologic changes in skin associated with aging”. J Dermatol Surg Oncol 16. 908-914. 1990.

[3] Yaar, M., Eller, M.S., and Gilchrest, B.A., "Fifty years of skin aging”. J Investig Dermatol Symp Proc 7. 51-58. 2002.

[4] Fisher, G.J., Kang, S., Varani, J., Bata-Csorgo, Z., Wan, Y., Datta, S., and Voorhees, J.J., "Mechanisms of photoaging and chronological skin aging”. Arch Dermatol 138. 1462-1470. 2002.

[5] Scharffetter-Kochanek, K., Brenneisen, P., Wenk, J., Herrmann, G., Ma, W., Kuhr, L., Meewes, C., and Wlaschek, M., "Photoaging of the skin from phenotype to mechanisms". Exp Gerontol 35. 307-316. 2000.

[6] Gilchrest, B.A., "Photoaging”. Nat Milest E2-E6. 2013.

[7] Ichihashi, M., Ueda, M., Budiyanto, A., Bito, T., Oka, M., Fukunaga, M., Tsuru, K., and Horikawa, T., "UV-induced skin damage”. Toxicology 189. 21-39. 2003.

[8] Reichrath, J., and Nurnberg, B., "Cutaneous vitamin D synthesis versus skin cancerdevelopment: The Janus faces of solar UVradiation”. Dermatoendocrinol 1. 253-261. 2009.

[9] Katiyar, S.K., Afaq, F., Perez, A., and Mukhtar, H., "Green tea polyphenol (-)-epigallocatechin-3-gallate treatment of human skin inhibits ultraviolet radiation-induced oxidative stress". Carcinogenesis 22. 287-294. 2001.

[10] Nichols, J.A., and Katiyar, S.K., "Skin photoprotection by natural polyphenols: anti-inflammatory, antioxidant and DNA repair mechanisms”. Arch Dermatol Res 302. 71-83. 2001.

[11] Qu, H., Zhang, Y., Chai, X., and Sun, W., "Isoforsythiaside, an antioxidant and antibacterial phenylethanoid glycoside isolated from Forsythia suspensa”. Bioorg Chem 40. 87-91. 2012.

[12] Piao, X.L., Jang, M.H., Cui, J., and Piao, X., "Lignans from the fruits of Forsythia suspensa”. Bioorg Med Chem Lett 18. 19801984. 2008.

[13] Lu, T., Piao, X.L., Zhang, Q., Wang, D., Piao, X.S., and Kim, S.W., "Protective effects of Forsythia suspensa extract against oxidative stress induced by diquat in rats”. Food Chem Toxicol 48. 764-770. 2010.

[14] Zhong, W.T., Wu, Y.C., Xie, X.X., Zhou, X., Wei, M.M., Soromou, L.W., Ci, X.X., and Wang, D.C., "Phillyrin attenuates LPS-induced pulmonary inflammation via suppression of MAPK and NF-kappaB activation in acute lung injury mice”. Fitoterapia 90. 132-139. 2013.

[15] Jiang, W.L., Tian, J.W., Fu, F.H., Zhu, H.B., and Hou, J., "Neuroprotective efficacy and therapeutic window of Forsythoside B: in a rat model of cerebral ischemia and reperfusion injury”. Eur J Pharmacol 640. 75-81. 2010.

[16] Cheng, G., Zhao, Y., Li, H., Wu, Y., Li, X., Han, Q., Dai, C.S., and $\mathrm{Li}$, Y.H., "Forsythiaside attenuates lipopolysaccharideinduced inflammatory responses in the bursa of Fabricius of chickens by downregulating the NF-kappaB signaling pathway". Exp Ther Med 7. 179-184. 2014.

[17] Douki, T., Reynaud-Angelin, A., Cadet, J., and Sage, E., "Bipyrimidine photoproducts rather than oxidative lesions are the main type of DNA damage involved in the genotoxic effect of solar UVA radiation”. Biochemistry 42. 9221-9226. 2003.

[18] Peres, P.S., Terra, V.A., Guarnier, F.A., Cecchini, R., and Cecchini, A.L., "Photoaging and chronological aging profile: Understanding oxidation of the skin”. J Photochem Photobiol B 103. 93-97. 2011. 
[19] Filip, A., Clichici, S., Daicoviciu, D., Catoi, C., Bolfa, P., Postescu, I. D., Gal, A., Baldea, I., Gherman, C., and Muresan, A., "Chemopreventive effects of Calluna vulgaris and Vitis vinifera extracts on UVB-induced skin damage in SKH-1 hairless mice”. $J$ Physiol Pharmacol 62. 385-392. 2011.

[20] Cadet J., and Douki, T., "Oxidatively generated damage to DNA by UVA radiation in cells and human skin". $J$ Invest Dermatol 131. 1005-1007. 2011.

[21] Darr D., and Fridovich, I., "Free radicals in cutaneous biology". $J$ Invest Dermatol 102. 671-675. 1994.

[22] Peus, D., Vasa, R.A., Meves, A., Pott, M., Beyerle, A., Squillace, K., and Pittelkow, M.R., "H2O2 is an important mediator of UVBinduced EGF-receptor phosphorylation in cultured keratinocytes". J Invest Dermatol 110. 966-971. 1998.

[23] Cortat, B., Garcia, C.C. M., Quinet, A., Schuch, A.P., de LimaBessa, K.M., and Menck, C.F.M., "The relative roles of DNA damage induced by UVA irradiation in human cells". Photochem Photobiol Sci 12. 1483-1495. 2013.

[24] Kunisada, M., Masaki, T., Ono, R., Morinaga, H., Nakano, E., Yogianti, F., Okunishi, K., Sugiyama, H., and Nishigori, C. "Hydrochlorothiazide enhances UVA-induced DNA damage". Photochem Photobiol 89. 649-654. 2013.

[25] Laat, A., Tilburg, M., Leun, J.C., Vloten, W.A., and Gruijl, F.R. "Cell cycle kinetics following UVA irradiation in comparison to UVB and UVC irradiation”. Photochem Photobiol 63. 492-497. 1996.
[26] Kastan, M.B., Onyekwere, O., Sidransky, D., Vogelstein, B., and Craig, R.W. "Participation of p53 protein in the cellular response to DNA damage”. Cancer Res 51. 6304-6311. 1991.

[27] Kuerbitz, S.J., Plunkett, B.S., Walsh, W.V., and Kastan, M.B. "Wild-type p53 is a cell cycle checkpoint determinant following irradiation”. Proc Natl Acad Sci U S A 89. 7491-7495. 1992.

[28] Ouhtit, A., Muller, H.K., Davis, D.W., Ullrich, S.E., McConkey, D., and Ananthaswamy, H.N., "Temporal events in skin injury and the early adaptive responses in ultraviolet-irradiated mouse skin". Am J Pathol 156. 201-207. 2000.

[29] Donoso, L.A., Kim, D., Frost, A., Callahan, A., annd Hageman, G. "The role of inflammation in the pathogenesis of age-related macular degeneration”. Surv Ophthalmol 51. 137-152. 2006.

[30] Bennett, M.F., Robinson, M.K., Baron, E.D., and Cooper, K.D., "Skin immune systems and inflammation: protector of the skin or promoter of aging?”. J Investig Dermatol Symp Proc 13. 15-19. 2008.

[31] Dudhgaonkar, S., Thyagarajan, A, and Sliva, D., "Suppression of the inflammatory response by triterpenes isolated from the mushroom Ganoderma lucidum”. Int Immunopharmacol 9. 12721280. 2009

[32] Jiang, W.L., Zhang, S.P., and Zhu, H.B. "Forsythoside B protects against experimental sepsis by modulating inflammatory factors". Phytother Res 26. 981-987. 2012.

[33] Reuter, S., Gupta, S.C., Chaturvedi, M.M., and Aggarwal, B.B., "Oxidative stress, inflammation, and cancer: how are they linked?”. Free Radic Biol Med 49. 1603-1616. 2010. 